Commun.Fac.Sci.Univ.Ank.Series A 1

Volume 56, Number 2, Pages 27-37 (2007)

ISSN $1303-5991$

\title{
EDGEWORTH SERIES APPROXIMATION FOR CHI-SQUARE TYPE CHANCE CONSTRAINTS
}

\author{
MEHMET YILMAZ
}

\begin{abstract}
We introduce two methods for approximation to distribution of weighted sum of chi-square random variables. These methods can be more useful than the known methods in literature to transform chi-square type chance constrained programming (CCP) problem into deterministic problem. Therefore, these are compared with Sengupta (1970)'s method. Some examples are illustrated for the purpose of comparing the solutions of these methods.
\end{abstract}

\section{INTRODUCTION}

The distribution of positive weighted combination of chi-square random variables with any degrees of freedom arises in many application areas such as communication theory, reliability of systems, engineering, industry etc. Many authors are interested in obtaining such as above distribution. Literature review of this subject was given by Johnson et al. (1994). Recent work of Castaño et al. (2005) derived a Laquerre expansion which has been used to evaluate distribution function of the sum of weighted central chi-square random variables. But it is more complicated for using stochastic programming. The best known example of a skewed sum is the chi-square distribution, of course chi-square distribution is itself asymptotically normal, thus this led us to approximate the distribution of a sum of weighted chi-square random variables by an expansion method based on central limit theorem (see Kendall, 1945; Patnaik, 1949; Feller, 1966 and Lehmann, 1999).

Next section can be viewed as recalling Castaño et al. (2005)'s work. Introduced methods will be compared with their work which can be seen a main tool of this paper for further discussions. In section three, we will give two methods based on normal approximation. These are called first and second edgeworth expansion respectively. We can adapt suggested methods to linear combination of independent random variables assumed to having finite fourth central moments. Chi-square

Received by the editors Sept. 28, 2007; Accepted: Dec. 25, 2007.

2000 Mathematics Subject Classification. Primary 62E20, 65K99; Secondary 60E07, 90C15.

Key words and phrases. weighted sum of chi-square random variables, expansion for distributions, chance constrained stochastic model. 
random variables have important role in many application areas of stochastic model. On the other hand, methods can be applied to solve CCP problem. In other words, it should be found their convenient deterministic equivalents to solve such a CCP problem. Two methods have some advantages of finding deterministic equivalents of chance constraints as these will be presented in fourth section.

\section{LAQUERRE EXPANSION}

Let $X_{1}, X_{2}, \ldots, X_{n}$ be independent chi-square random variables with $v_{i}(i=1,2, \ldots, n)$ degrees of freedom, respectively. Consider $\sum_{i=1}^{n} a_{i} X_{i}$, where $a_{i}>0$. Distribution of $\sum_{i=1}^{n} a_{i} X_{i}$ as a Laquerre series expansion is given by Castaño et al. (2005) as follows:

$$
F(x)=\frac{e^{-\frac{x}{2 \beta}}}{(2 \beta)^{1+\frac{v}{2}}} \frac{x^{\frac{v}{2}}}{\Gamma\left(\frac{v}{2}+1\right)} \sum_{k=0}^{\infty} \frac{k ! m_{k}}{\left(\frac{v}{2}+1\right)_{k}} L_{k}^{\left(\frac{v}{2}\right)}\left(\frac{(v+2) x}{4 \beta \mu_{0}}\right)
$$

with

$v=\sum_{i=1}^{n} v_{i}, p=\frac{v}{2}+1$

and

$m_{k}=\frac{1}{k} \sum_{j=0}^{k-1} m_{j} I_{k-j}, k \geq 1$, where $m_{0}=\left(\frac{p}{\mu_{0}}\right)^{\frac{v}{2}} \frac{2 \beta p}{p-\mu_{0}} \prod_{i=1}^{n}\left(1+\frac{a_{i}}{\beta}\left(\frac{p}{\mu_{0}}-1\right)\right)^{-\frac{v_{i}}{2}}$

and

$$
I_{j}=\left(\frac{-1}{\frac{p}{\mu_{0}}-1}\right)^{j}+\frac{1}{2} \sum_{i=1}^{n} v_{i}\left(\frac{1-\frac{a_{i}}{\beta}}{1+\frac{a_{i}}{\beta}\left(\frac{p}{\mu_{0}}-1\right)}\right)^{j}, j \geq 1
$$

where $L_{k}^{\left(\frac{v}{2}\right)}()=.\sum_{r=0}^{k}\left(\begin{array}{c}r+v / 2 \\ k-r\end{array}\right) \frac{(.)^{r}}{r !}$, is the generalized Laguerre polynomial. They have obtained the following bound for the truncation error of the distribution function $F$

$$
\varepsilon_{N} \leq \frac{e^{-\frac{x}{2 \beta}}}{(2 \beta)^{1+\frac{v}{2}}} \frac{x^{\frac{v}{2}}\left|m_{0}\right|}{\Gamma\left(\frac{v}{2}+1\right)} \exp \left(\frac{(v+2) x}{8 \beta \mu_{0}}\right) \sum_{k=N}^{\infty} \frac{\left(\frac{v}{2}+1\right)_{k}}{k !} \xi^{k}
$$

where $\xi=\max _{i}\left|\frac{1-\frac{a_{i}}{\beta}}{1+\frac{a_{i}}{\beta}\left(\frac{p}{\mu_{0}}-1\right)}\right|$. This expansion converges uniformly in any finite interval, for all $\mu_{0}$ and $\beta$ conveniently chosen. They also showed with illustrative examples that if $\mu_{0}=p / 10$ and $\beta=\left(a_{1}+a_{n}\right) / 2$ are chosen, then truncation error bound is quite small. Therefore our results will be compared with each other for the same values of $\mu_{0}$ and $\beta$. 


\section{EXPANSION FOR DISTRIBUTIONS}

In this section, we will introduce two known expansions for sum of independent and identical random variables related to the central limit theorem. These are called as first and second edgeworth expansion in the literature. First edgeworth expansion which is well known yields more approximated probability than normal approximation does. Also, second edgeworth expansion could be better than the first for the sum of chi-square random variables (see Kendall, 1945; Patnaik, 1949 and Lehmann, 1999). Hence, extensions of two methods for the weighted chi-square random variables, are set up the following subsection.

Theorem 3.1. Let $X_{1}, X_{2}, \ldots, X_{n}$ be independent continuous random variables such that $E\left|X_{j}\right|^{3}<\infty(j=1,2, \ldots, n)$, for large values of $n$, then

$$
P\left(\frac{\sum_{j=1}^{n} X_{j}-E\left(\sum_{j=1}^{n} X_{j}\right)}{\left(\mu_{2}^{(n)}\right)^{\frac{1}{2}}} \leq x\right)=\Phi(x)+\frac{\mu_{3}^{(n)}\left(1-x^{2}\right)}{6\left(\mu_{2}^{(n)}\right)^{\frac{3}{2}}} \phi(x)+o\left(\frac{n}{\left(\mu_{2}^{(n)}\right)^{\frac{3}{2}}}\right)
$$

is used approximation to standard normal distribution. Here $\Phi$ and $\phi$ stand for standard normal cumulative distribution function, its density function respectively and $\mu_{k}^{(n)}$ denotes $\sum_{j=1}^{n} E\left(X_{j}-E X_{j}\right)^{k}$ (Feller, 1966 Chapter XVI).

The first term given in (2) is known as first term edgeworth expansion in the literature. The second term edgeworth expansion can be defined without loss of generality as follows:

Subtract from the right side of (2) the term

$$
\left[\frac{K_{4}}{24}\left(x^{3}-3 x\right)+\frac{K_{3}^{2}}{72}\left(x^{5}-10 x^{3}+15 x\right)\right] \phi(x)
$$

where $K_{3}$ and $K_{4}$ are third and fourth cumulants of $\frac{\sum_{j=1}^{n}\left(X_{j}-E\left(X_{j}\right)\right)}{\sqrt{\sum_{j=1}^{n}\left(X_{j}-E\left(X_{j}\right)\right)^{2}}}$ (see Wallace,

1958). If the random variables are identically distributed then the quantity given in (3) can be written as

$$
\left[\frac{\mu_{4}^{(n)}-\frac{3\left(\mu_{2}^{(n)}\right)^{2}}{24\left(\mu_{2}^{(n)}\right)^{2}}}{2}\left(x^{3}-3 x\right)+\frac{\left(\mu_{3}^{(n)}\right)^{2}}{72\left(\mu_{2}^{(n)}\right)^{3}}\left(x^{5}-10 x^{3}+15 x\right)\right] \phi(x) .
$$


3.1. Set-up for Weighted Chi-Square Random Variables. Consider random variables $X_{i}(i=1,2, \ldots, n)$ distributed as chi-square with $v_{i}(i=1,2, \ldots, n)$ degrees of freedom. The probability density function of $X_{i}(i=1,2, \ldots, n)$ is given by

$$
f\left(x_{i} ; v_{i}\right)=\frac{1}{\Gamma\left(\frac{v_{i}}{2}\right) 2^{\frac{v_{i}}{2}}} x_{i}^{\frac{v_{i}}{2}-1} \exp \left(-\frac{x_{i}}{2}\right), x_{i}>0, v_{i}>0(i=1,2, \ldots, n) .
$$

The central moments $\mu_{l}^{(1)}(l=1,2,3,4)$ can be obtained by evaluating the following integral,

$$
\begin{aligned}
\mu_{k}^{(1)} & =\frac{1}{\Gamma\left(\frac{v_{i}}{2}\right) 2^{\frac{v_{i}}{2}}} \int_{0}^{\infty}\left(x-v_{i}\right)^{k} x^{\frac{v_{i}}{2}-1} \exp \left(-\frac{x}{2}\right) d x \\
& =\sum_{j=0}^{k}\left(\begin{array}{c}
k \\
j
\end{array}\right) \frac{\left(-v_{i}\right)^{k-j} \Gamma\left(\frac{v_{i}}{2}+j\right) 2^{j}}{\Gamma\left(\frac{v_{i}}{2}\right)}, \quad k=1,2,3, \ldots
\end{aligned}
$$

Hence $\mu_{1}^{(1)}=0, \mu_{2}^{(1)}=2 v_{i}, \mu_{3}^{(1)}=8 v_{i}$ and $\mu_{4}^{(1)}=12 v_{i}^{2}+48 v_{i}$ are calculated. From now on, we can set up (2) and (3) for the weighted sum of chi-square variables. Let

$$
M_{1}=\sum_{i=1}^{n} a_{i} v_{i}, M_{2}=2 \sum_{i=1}^{n} a_{i}^{2} v_{i}, M_{3}=8 \sum_{i=1}^{n} a_{i}^{3} v_{i}, M_{4}=48 \sum_{i=1}^{n} a_{i}^{4} v_{i}
$$

$M_{k}(k \geq 1)$ denote cumulants of $\sum_{i=1}^{n} a_{i}\left(X_{i}-E\left(X_{i}\right)\right)$, then $P\left(\sum_{i=1}^{n} a_{i} X_{i} \leq t\right)$ is approximated as

$$
\begin{gathered}
\approx \Phi(x)+\frac{M_{3}\left(1-x^{2}\right)}{6\left(M_{2}\right)^{\frac{3}{2}}} \phi(x) \\
\approx \Phi(x)+\left[\frac{M_{3}\left(1-x^{2}\right)}{6 M_{2}^{\frac{3}{2}}}-\frac{M_{4}\left(x^{3}-3 x\right)}{24 M_{2}^{2}}-\frac{M_{3}^{2}\left(x^{5}-10 x^{3}+15 x\right)}{72 M_{2}^{3}}\right] \phi(x) \\
\approx \Phi(x)+\left[\frac{M_{3}\left(1-x^{2}\right)}{6 M_{2}^{\frac{3}{2}}}-\frac{\left(M_{4}^{\prime}-\frac{3 M_{2}^{2}}{n}\right)\left(x^{3}-3 x\right)}{24 M_{2}^{2}}-\frac{M_{3}^{2}\left(x^{5}-10 x^{3}+15 x\right)}{72 M_{2}^{3}}\right] \phi(x)
\end{gathered}
$$

where $x=\frac{t-M_{1}}{\sqrt{M_{2}}}$, and $M_{4}^{\prime}=\sum_{i=1}^{n} a_{i}^{4}\left(12 v_{i}^{2}+48 v_{i}\right)$. First and second edgeworth approximations for distribution of weighted sum of chi-square random variables can be given respectively in (6) and (7). Here the second expression $\left(7^{*}\right)$ can be viewed as modified and extended version for weighted sum, this modified expansion allows to get better results than the other when the chi-square random variables have different degrees of freedom. Otherwise, $(7)$ and $\left(7^{*}\right)$ give same result for $v_{1}=v_{2}=\ldots=v_{n}$.

In Table 1, we first compare our results for $P\left(2 \chi_{(1)}^{2}+2 \chi_{(1)}^{2} \leq t\right), n=2$ and $a_{1}=a_{2}, v_{1}=v_{2}$. Here FE, SE and LE denote first term edgeworth expansion, second term edgeworth expansion and Laquerre expansion. These are calculated from Matlab. 
Notes: (i) Since the weights are equal and random variables are independent and identically distributed, if the quantity $P\left(2 \chi_{(1)}^{2}+2 \chi_{(1)}^{2} \leq t\right)$ can be arranged as $P\left(\chi_{(2)}^{2} \leq \frac{t}{2}\right)$, then it is easy to compare FE and SE by checking chi-square tables. Table 1 and Table 3 are specially presented because of these probabilities are easily checked from chi-square tables. So that tabulations could be useful to find an answer how close FE and SE to exact values.

(ii) Various approximation methods of distribution of weighted sum of chi-square random variables can be seen in Johnson et al. (1994) (see Chapter 8 pp. 444), but most of these methods is stated by infinite series like as laquerre expansion. Therefore, although these methods perform to get better results on calculating such probabilities, it is difficult to apply them to solve CCP problem.

TABle 1. Computed probability for $n=2$

\begin{tabular}{|c|c|c|c|c|c|c|c|}
\hline & \multicolumn{3}{|c|}{ Probability } & & \multicolumn{3}{c|}{ Probability } \\
\hline $\mathrm{t}$ & $\mathrm{FE}$ & $\mathrm{SE}$ & Table & $\mathrm{t}$ & $\mathrm{FE}$ & $\mathrm{SE}$ & Table \\
\hline 1 & .270543 & .254516 & .221199 & 14 & .963116 & .948052 & .969803 \\
\hline 2 & .396554 & .398387 & .393469 & 15 & .977128 & .953374 & .976482 \\
\hline 3 & .522127 & .528358 & .527633 & 16 & .986832 & .962457 & .981684 \\
\hline 4 & .632981 & .632981 & .632120 & 17 & .992955 & .972816 & .985736 \\
\hline 5 & .719540 & .713310 & .713495 & 18 & .996495 & .982209 & .988891 \\
\hline 6 & .779478 & .777645 & .776870 & 19 & .998376 & .989421 & .991348 \\
\hline 7 & .817288 & .833316 & .826226 & 20 & .999299 & .994258 & .993262 \\
\hline 8 & .841345 & .881673 & .864665 & 21 & .999718 & .997144 & .994752 \\
\hline 9 & .860103 & .919114 & .894601 & 22 & .999894 & .998694 & .995913 \\
\hline 10 & .879227 & .941962 & .917915 & 23 & .999963 & .999450 & .996817 \\
\hline 11 & .900625 & .950659 & .936072 & 24 & .999988 & .999786 & .997521 \\
\hline 12 & .923259 & .950254 & .950213 & 25 & .999996 & .999923 & .998069 \\
\hline 13 & .944795 & .947631 & .961226 & 26 & .999998 & .999974 & .998496 \\
\hline
\end{tabular}


For different degrees of freedom and different weights with two components, the quantity of $P\left(1.4 \chi_{(2)}^{2}+3.3 \chi_{(3)}^{2} \leq t\right)$ is computed four methods and given in Table 2. Here MSE stands for modified second term edgeworth expansion.

TABle 2. Computed probability for different weights and different d.f.

\begin{tabular}{|l|c|c|c|c|c|c|c|c|c|}
\hline$t$ & \multicolumn{4}{|c|}{ Probability } & $t$ & \multicolumn{5}{c|}{ Probability } \\
\hline & FE & SE & MSE & LE & & FE & SE & M SE & LE \\
\hline 1 & .052810 & .018692 & .009360 & .005370 & 20 & .821447 & .837584 & .858319 & .796862 \\
\hline 2 & .080648 & .048156 & .035514 & .025903 & 23 & .864742 & .896060 & .909979 & .840833 \\
\hline 3 & .113955 & .084896 & .069164 & .061071 & 26 & .899254 & .931859 & .936024 & .869576 \\
\hline 4 & .152607 & .128397 & .110049 & .107597 & 29 & .930412 & .949092 & .946081 & .888062 \\
\hline 5 & .196217 & .177708 & .157473 & .161835 & 32 & .957071 & .957580 & .951884 & .899791 \\
\hline 8 & .349154 & .346375 & .326752 & .340898 & 35 & .976768 & .965710 & .960616 & .907145 \\
\hline 11 & .511898 & .513718 & .504839 & .505808 & 38 & .989046 & .975693 & .972394 & .911648 \\
\hline 14 & .653359 & .651804 & .658688 & .636213 & 41 & .995507 & .985435 & .983735 & .914189 \\
\hline 17 & .755804 & .757199 & .775934 & .731156 & & & & & \\
\hline
\end{tabular}

Now, it is assumed that for $n=4$ with equal weights and equal d.f., the quantity of $P\left(2 \chi_{(1)}^{2}+2 \chi_{(1)}^{2}+2 \chi_{(1)}^{2}+2 \chi_{(1)}^{2} \leq t\right)$ is tabulated and given Table 3 .

TABle 3. Computed probability for equal weights and equal d.f.

\begin{tabular}{|l|c|c|c|c|c|c|c|}
\hline & \multicolumn{3}{|c}{ Probability } & \multicolumn{3}{c|}{ Probability } \\
\hline$t$ & FE & SE & Table & $t$ & FE & SE & Table \\
\hline 1 & 0.084731 & 0.055542 & 0.026499 & 19 & 0.934601 & 0.950864 & 0.950253 \\
\hline 3 & 0.202297 & 0.187806 & 0.173358 & 21 & 0.960512 & 0.959886 & 0.967203 \\
\hline 5 & 0.356660 & 0.356825 & 0.355364 & 23 & 0.979135 & 0.968419 & 0.978516 \\
\hline 7 & 0.519523 & 0.522086 & 0.522122 & 25 & 0.990414 & 0.978251 & 0.986004 \\
\hline 9 & 0.659839 & 0.657276 & 0.657452 & 27 & 0.996176 & 0.987358 & 0.990926 \\
\hline 11 & 0.760777 & 0.760613 & 0.760270 & 29 & 0.998675 & 0.993836 & 0.994141 \\
\hline 13 & 0.825538 & 0.840030 & 0.835210 & 31 & 0.999600 & 0.997470 & 0.996231 \\
\hline 15 & 0.868807 & 0.897996 & 0.888291 & 33 & 0.999895 & 0.999120 & 0.997583 \\
\hline 17 & 0.903581 & 0.933492 & 0.925113 & 35 & 0.999976 & 0.999740 & 0.998455 \\
\hline
\end{tabular}

We will show the results for $P\left(3.1 \chi_{(1)}^{2}+2.9 \chi_{(2)}^{2}+4.3 \chi_{(3)}^{2}+1.7 \chi_{(4)}^{2} \leq t\right)$ (Different weights, different d.f. for $n=4$ ) in Table4. 
TABle 4. Computed probability for different weights and different d.f.

\begin{tabular}{|l|c|c|c|c|c|c|c|c|c|}
\hline $\mathrm{t}$ & \multicolumn{4}{|c|}{ Probability } & $\mathrm{t}$ & \multicolumn{4}{|c|}{ Probability } \\
\hline & $\mathrm{FE}$ & $\mathrm{SE}$ & $\mathrm{MSE}$ & $\mathrm{LE}$ & & $\mathrm{FE}$ & $\mathrm{SE}$ & $\mathrm{MSE}$ & $\mathrm{LE}$ \\
\hline 6 & .018190 & .002247 & .001344 & .005749 & 17 & .211790 & .202263 & .191458 & .195038 \\
\hline 8 & .037324 & .019293 & .016464 & .017641 & 20 & .299419 & .295326 & .284566 & .285651 \\
\hline 10 & .063205 & .044634 & .039674 & .039390 & 25 & .459163 & .459568 & .453601 & .437698 \\
\hline 13 & .115891 & .099525 & .091436 & .092387 & 30 & .609774 & .609422 & .611860 & .568382 \\
\hline 15 & .160379 & .147097 & .137336 & .140013 & & & & & \\
\hline
\end{tabular}

We can see that for $n=2$, for smaller values of $t$, the MSE is closer to Laquerre expansion, on the contrary, for larger values of $t$, first term expansion gets better results. For $n=4, \mathrm{SE}$ and MSE are almost better than FE.

\section{Chance Constrained Stochastic Model}

Chance constrained programming method was originally introduced by Charnes and Cooper (1959). One of the common problems in the practical application of mathematical programming is the difficulty of determining the proper values of the model coefficients. The true values of these coefficients may not become known until after a solution has been chosen and implemented. This can sometimes be attributed solely to the inadequacy of the investigation. However, the values of these coefficients take on often are influenced by random events that impossible to predict. In short, some or all of the model coefficients may be random variables. We will introduce simple stochastic model with one probabilistic constraint as follows:

$$
\begin{gathered}
\max (\min ) z(a)=g(a), \\
P\left(\sum_{i=1}^{n} a_{i} X_{i} \leq t\right) \geq 1-\alpha, \\
a_{i} \geq 0, i=1,2, \ldots, n, \\
\alpha \in(0,1) .
\end{gathered}
$$

where $\alpha$ is the specified probability. Here, it is assumed that $a_{i}(i=1,2, \ldots, n)$ decision variables are deterministic, technologic coefficients; $X_{i}(i=1,2, \ldots, n)$ are random variables. Thus, the problem is to find deterministic equivalent of probabilistic constraint. On the purpose of this paper, we consider the chance constrained stochastic programming problem when the $X_{i}$ 's are random variables distributed as chi-square. The equivalent deterministic problem is obtained by three methods. First two methods are related to first term and second term edgeworth expansion introduced previous section. Third method offered by Sengupta (1970) can be easily 
transform to (8) into its deterministic equivalent. Such that, $P\left(\sum_{i=1}^{n} a_{i} X_{i} \leq t\right) \geq 1-\alpha$ is equivalent to

$$
t\left(\sum_{i=1}^{n} a_{i} E\left(X_{i}\right)\right)-\chi_{(v)}^{2^{i n v}}\left(\sum_{i=1}^{n} a_{i}^{2} E\left(X_{i}\right)\right) \geq 0
$$

Here $E\left(X_{i}\right)=v_{i}, v=\sum_{i=1}^{n} v_{i}$ and $\chi_{(v)}^{2^{i n v}}$ denotes inverse distribution function with $v$ degrees of freedom on the $1-\alpha$ level.

We will compare values of the objective function of model, which are obtained from these three methods by giving numerical examples, and we will discuss on these results by illustrating examples in this section. We will also compare three methods with Laguerre expansion introduced by Castaño et al. (2005), since their tool still gives more appropriate result.

Example 4.1. For $n=2$, here is simple stochastic model with one chance constraint, it is assumed that the random variables have the same chi-square distribution with degrees of freedom 1 and independent:

$$
\begin{gathered}
\max z=a_{1}+a_{2} \\
P\left(a_{1} \chi_{(1)}^{2}+a_{2} \chi_{(1)}^{2} \leq 4\right) \geq 0.6 \\
1 \leq a_{1}, a_{2} \leq 6 .
\end{gathered}
$$

Solutions have been obtained by using Lingo 9.0. and presented in Table 5 .

TABLE 5. Model solutions for (10)

\begin{tabular}{|l|cl|l|}
\hline $\mathrm{t}=4$ & \multicolumn{2}{|c|}{ Decision Variables $(a)$} & Objective Function $(z)$ \\
\hline $\mathrm{FE}$ & $a_{1}=3.977762$ & $a_{2}=1.000000$ & 4.977762 \\
\hline $\mathrm{MSE}$ & $a_{1}=3.909999$ & $a_{2}=1.000000$ & 4.909999 \\
\hline $\mathrm{SM}$ & $a_{1}=2.182713$ & $a_{2}=2.182713$ & 4.365427 \\
\hline
\end{tabular}

Even if Lingo Program yields to global solutions for three methods on the probability level at 0.6 , the solutions are checked by using Laquerre expansion method, except for SM (Sengupta's method), FE and SE are not greater than 0.6. Furthermore, among the three methods, when they are compared with each other according to their objective function values, SM has the least value. But Laquerre expansion shows that the solution of the model approximately is $a_{1}=3.825538$ and $a_{2}=1$, and the objective function value is 4.825538. In this situation, SE and FE methods give better result than SM method does. It can be emphasized that even if $\mathrm{FE}$ and 
SE are related to large sample theory, these can also perform to get better results for two weighted components.

Example 4.2. For $n=2$, suppose that random variables are independent but not identically distributed, then the model is setting up as follows:

$$
\begin{gathered}
\max z=a_{1}+a_{2}, \\
P\left(a_{1} \chi_{(2)}^{2}+a_{2} \chi_{(4)}^{2} \leq t\right) \geq 0.6, \\
a_{1}, a_{2} \geq 1 .
\end{gathered}
$$

Solutions have been obtained for some values of $t$ and given in Table 6 .

TABLE 6. Model solutions for (11)

\begin{tabular}{|l|l|ll|l|}
\hline & & \multicolumn{2}{|c|}{ Decision variables $(a)$} & Objective Function $(z)$ \\
\hline & $\mathrm{FE}$ & $a_{1}=1.399968$ & $a_{2}=1.000000$ & 2.399968 \\
\hline $\mathrm{t}=7$ & $\mathrm{MSE}$ & $a_{1}=1.397556$ & $a_{2}=1.000000$ & 2.397556 \\
\hline & $\mathrm{SM}$ & $a_{1}=1.319666$ & $a_{2}=1.000000$ & 2.319666 \\
\hline & $\mathrm{FE}$ & $a_{1}=1.935567$ & $a_{2}=1.000000$ & 2.935567 \\
\hline $\mathrm{t}=8$ & $\mathrm{MSE}$ & $a_{1}=1.886886$ & $a_{2}=1.019945$ & 2.906831 \\
\hline & $\mathrm{SM}$ & $a_{1}=1.554859$ & $a_{2}=1.099452$ & 2.654311 \\
\hline & $\mathrm{FE}$ & $a_{1}=2.491195$ & $a_{2}=1.000000$ & 3.491195 \\
\hline $\mathrm{t}=9$ & $\mathrm{MSE}$ & $a_{1}=2.122746$ & $a_{2}=1.147438$ & 3.270185 \\
\hline & $\mathrm{SM}$ & $a_{1}=1.749217$ & $a_{2}=1.236883$ & 2.986100 \\
\hline & $\mathrm{FE}$ & $a_{1}=3.053301$ & $a_{2}=1.000000$ & 4.053301 \\
\hline $\mathrm{t}=10$ & $\mathrm{MSE}$ & $a_{1}=3.049645$ & $a_{2}=1.000000$ & 4.049645 \\
\hline & $\mathrm{SM}$ & $a_{1}=1.943574$ & $a_{2}=1.374315$ & 3.317889 \\
\hline
\end{tabular}

We can see from Table 6 that FE and MSE yield better solutions than SM, according to their objective function values.

Example 4.3. For $n=4$, random variables are identically and independently distributed, and model has one chance constraint and some deterministic constraints,

$$
\begin{gathered}
\max z=a_{1} a_{2} a_{3} a_{4}, \\
P\left(a_{1} \chi_{(1)}^{2}+a_{2} \chi_{(1)}^{2}+a_{3} \chi_{(1)}^{2}+a_{4} \chi_{(1)}^{2} \leq t\right) \geq 0.6, \\
2 a_{2}+4 a_{4} \leq 20 \\
5 a_{1}+3 a_{3} \leq 15 \\
a_{1} a_{4} \geq 4 \\
a_{2} \leq 5 \\
a_{3} \geq 2 .
\end{gathered}
$$

Solutions have been obtained for some values of $t$ and given in Table 7 . 
TABle 7. Model solutions for (12)

\begin{tabular}{|c|c|c|c|c|}
\hline & & \multicolumn{2}{|c|}{ Decision variables $(a)$} & $\begin{array}{c}\text { Objective } \\
\text { Function }(z)\end{array}$ \\
\hline & $\mathrm{FE}$ & $\begin{array}{c}a_{1}=1.800000 \\
a_{2}=0.9968352\end{array}$ & $\begin{array}{l}a_{3}=2.000000 \\
a_{4}=2.222222\end{array}$ & 7.974682 \\
\hline \multirow[t]{3}{*}{$\mathrm{t}=7$} & $\mathrm{SE}$ & $\begin{array}{c}a_{1}=1.800000 \\
a_{2}=0.9971481\end{array}$ & $\begin{array}{l}a_{3}=2.000000 \\
a_{4}=2.222222\end{array}$ & 7.977185 \\
\hline & SM & solution can not & be found & \\
\hline & $\mathrm{FE}$ & $\begin{array}{l}a_{1}=1.800000 \\
a_{2}=1.905983\end{array}$ & $\begin{array}{l}a_{3}=2.000000 \\
a_{4}=2.222222\end{array}$ & 15.24786 \\
\hline \multirow[t]{3}{*}{$t=8$} & $\mathrm{SE}$ & $\begin{array}{l}a_{1}=1.800000 \\
a_{2}=1.903263\end{array}$ & $\begin{array}{l}a_{3}=2.000000 \\
a_{4}=2.222222\end{array}$ & 15.22610 \\
\hline & $\mathrm{SM}$ & $\begin{array}{l}a_{1}=1.800000 \\
a_{2}=1.832373\end{array}$ & $\begin{array}{l}a_{3}=2.000000 \\
a_{4}=2.222222\end{array}$ & 14.65899 \\
\hline & $\mathrm{FE}$ & $\begin{array}{l}a_{1}=1.773540 \\
a_{2}=3.103829\end{array}$ & $\begin{array}{l}a_{3}=2.044100 \\
a_{4}=3.103829\end{array}$ & 34.92518 \\
\hline \multirow[t]{2}{*}{$\mathrm{t}=10$} & $\mathrm{SE}$ & $\begin{array}{l}a_{1}=1.773841 \\
a_{2}=3.104133\end{array}$ & $\begin{array}{l}a_{3}=2.043599 \\
a_{4}=3.104133\end{array}$ & 34.92937 \\
\hline & $\mathrm{SM}$ & $\begin{array}{l}a_{1}=1.733264 \\
a_{2}=2.833099\end{array}$ & $\begin{array}{l}a_{3}=2.111227 \\
a_{4}=2.833099\end{array}$ & 29.37129 \\
\hline
\end{tabular}

From Table 7, it is possible to say that using transformed chance constraint(s) into its (their) deterministic equivalent(s) by constructing with FE or SE in a model, one can obtain better solution using Lingo Program.

\section{Conclusion}

In this paper, we have suggested two expansions based on normal approximation for weighted sum of chi-square random variables. These are applied to one of the specific stochastic model which is a chance constrained programming problem. It is assumed that a decision variables are deterministic, technologic coefficients are independent chi-square random variables. It is aimed to transform the chance constraints into deterministic constraints by using these methods. We compare the solutions of transformed deterministic model according to objective functions. As it can be seen from Table 5, Table 6 and Table 7, FE and SE yield more feasible results than SM method. If the random variables have chi-square distribution with different d.f., then FE and SE result's are closely feasible. Therefore FE and SE are recommended to converting chance constraint into deterministic constraint for chi-square type CCP problem. 


\section{Kİ-KARE TİPİ ŞANS KISITLARI İÇİN EDGEWORTH SERİ YAKLAŞIMI}

ÖZET: Ki-kare dağılımlı rasgele değişkenlerin ağırlıklı toplamının dağılımına yaklaşım için iki yöntem tanıtılmıştır. Öyle ki bu yöntemler, ki-kare tipindeki şans kısıtlı problemlerin deterministik eşitliklerinin elde edilmesinde kullanılabilir ve literatürde bilinen yöntemlerden daha yararlı olabilir. Bu nedenle, bu iki limit yaklaşımı, Sengupta (1970)' nın bu tür problemler için önerdiği yöntem ile karşılaştırılmış ve bu amaç doğrultusunda bazı örnekler verilmiştir.

Acknowledgments: I wish to thank the referees for their valuable comments.

\section{REFERENCES}

[1] Castaño, M.A., and López, B.F., Distribution of a Sum of Weighted Central Chi- Squared Variables. Communications in Statistics-Theory and Methods, vol. 34; (2005), pp. 515-524.

[2] Charnes A. and Cooper W. W., Chance-Constrained Programming. Management Sci. 5: (1959), pp. 73-79.

[3] Feller, W., An Introduction to Probability Theory and Its Applications, Volume II, 1966, John Wiley and Sons, Inc. New York, London.

[4] Johnson, N.L., Kotz, S., and Balakrishnan, N., Continuous Univariate Distributions I, Second Ed., 1994, New York, John Wiley and Sons.

[5] Kendall, M.G., The Advanced Theory of Statistics, Volume I, 1945, Charles Griffin Company Limited.

[6] Lehmann, E.L., Elements of Large Sample Theory, 1999, Springer Verlag, New York Inc.

[7] Patnaik, P.B., The Non Central Chi-Square and F-Distribution and Their Applications. Biometrika, vol.36; No:1/2, (1949), pp.202-232.

[8] Sengupta, J.K. A., Generalization of Some Distribution Aspects of Chance Constrained Linear Programming. International Economic Review, vol. 11, (1970), pp. 287-304.

[9] Wallace, D. L., Asymptotic Approximations to Distributions. The Annals of Mathematical Statistics, Vol. 29, No. 3, (1958), pp. 635-654.

Current address: Ankara University, Faculty of Sciences, Department of Statistics, 06100 Tandoğan-Ankara, Turkey

E-mail address: yilmazm@science.ankara.edu.tr 